\title{
Design of Daisy-chain Connection for Flexible and Scalable Ultrasonic Sensor Array
}

\author{
Yuichi Morita ${ }^{\mathrm{a}}$, Akira Yamawaki ${ }^{\mathrm{a}}$ \\ ${ }^{a}$ Kyushu Institute of Technology, Kitakyushu, 804-8550 Japan \\ *Corresponding Author: yama@ecs.kyutech.ac.jp
}

\begin{abstract}
Non-destructive inspection using ultrasonic sensor array has been widely employed for various objects to detect internal flaws or to characterize materials. The measured objects have different size and shape, so we need to move sensor array along the surface mechanically. This way takes a lot of time to inspect the objects. To solve this problem, we have proposed the conceptual organization of ultrasonic sensor array with scalability and flexibility to any objects to be measured. This paper describes the daisy-chain connection to realize the proposed ultrasonic sensor array in detail. In this structure, several ultrasonic sensor modules that can be adhered to the measured objects are connected by serial communication with flexible connectors can be bending in a daisy chain fashion. If measured objects are huge or the shape is complex, many modules are needed to cover the object surface efficiently. This may cause delay to complete measuring at all modules because outputs of each module are sent to the host PC sequentially. As a preliminary evaluation for the proposed structure, we investigate the relation between the number of the modules and transfer rate from modules to the host computer.
\end{abstract}

Keywords: ultrasonic sensor array, flexibility, scalability.

\section{Introduction}

Ultrasonic sensors are used in many fields and applications such as detection of target objects and measurement of distance and so on. The measured objects have different size and shape, so we need to move sensors along the surface mechanically. This way takes a lot of time to inspect the objects. Previous studies tried to solve this problem in various ways. As one of them, a flexible thin-film ultrasonic sensor is reported ${ }^{(1)}$. This sensor can change its
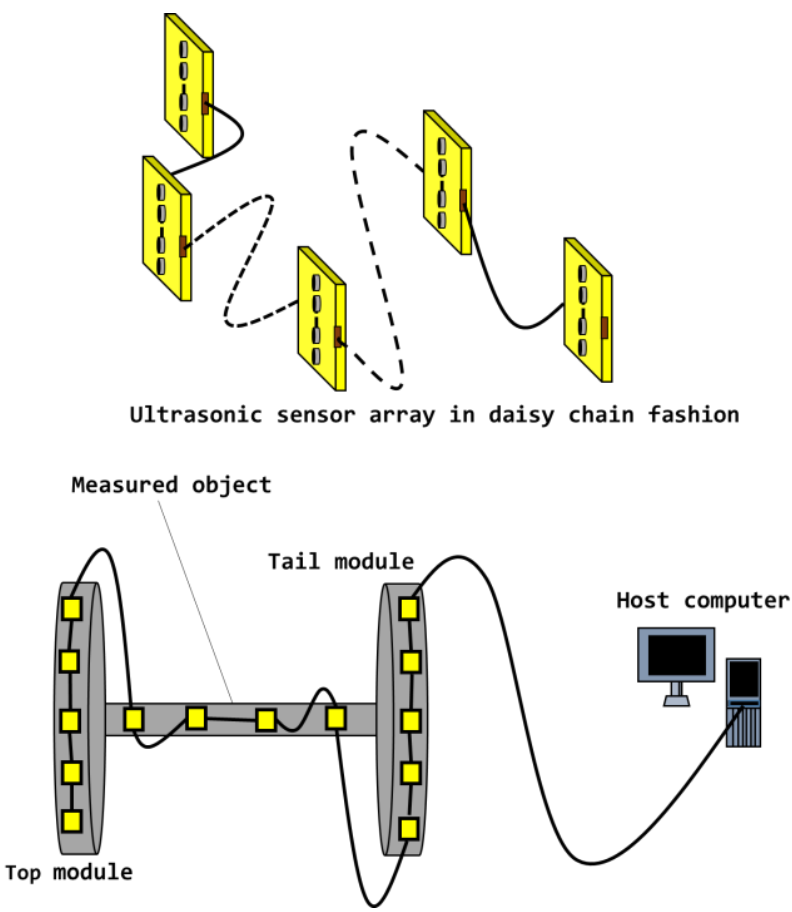

Fig. 1. Proposed Ultrasonic Sensor Array

form according to the shape of the measured objects. However, we need to manufacture new sensors that fit measured objects. This problem would cause increase in cost. Ultrasonic sensor array that arranges many ultrasonic sensors have been developed to save time to scan measured objects and improve resolution. As one of them, captive micromachined ultrasonic transducer (CMUT) is developed $^{(2)}$. Using CMUT, many ultrasonic transducers can be intergraded and connected with electronics compactly. However, the more number of elements increases, the more number of cables increases. This makes the instrument complex ${ }^{(3)}$.

To solve these problems, we have proposed the conceptual organization of ultrasonic sensor array with scalability and flexibility to any objects ${ }^{(4)}$. As shown in Fig. 1 , arbitrary number of ultrasonic sensor modules that are able 
to adhere to the measured objects are connected in a daisy chain fashion. Each module is connected by flexible cables, so the whole of the instrument can fit arbitrary objects that have various shape and size. Each module is controlled by the host computer. The module connected to the host computer is named "tail module" and the module that is end of the array is named "top module". The signal indicating the beginning of the measuring is passed through via serial communication from the tail module to the top module sequentially. When the modules receive the signal, measuring is performed and the data is sent to the host computer from the top module to the tail module.

In this paper, we first explain the organization of the proposed ultrasonic sensor array. In this structure, modules equipped with several ultrasonic transducers that operate as speaker and sensor and processing units are connected in daisy chain fashion. We describe the operation in the whole of the instrument and the module to realize the proposed ultrasonic sensor array. The ultrasonic sensor array is composed of any ultrasonic sensor modules and the host computer that controls each module. Communication among the host computer and each module is performed by serial communication. So delay would be concerned if the large number of modules are connected. As a preliminary evaluation, we investigate the relation between the number of the modules and transfer rate from modules to the host computer.

\section{Ultrasonic Sensor Module}

\subsection{Measuring Method of Distance}

In our structure, outputs of ultrasonic sensors are processed as binary data. The " 1 " of bit means that reflected wave has arrived to the sensors. The " 0 " of bit means that reflected wave has never arrived yet. As shown in Fig. 2, outputs of ultrasonic sensors in binary data are saved into the memory operates as data logger at a certain time. Distance can be calculated by multiplying the time when the " 1 " of bit is gotten with sound speed.

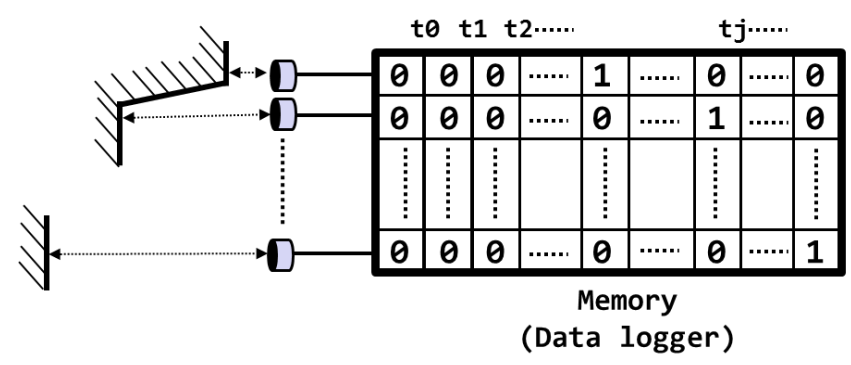

Fig. 2. Outputs of Ultrasonic Sensors

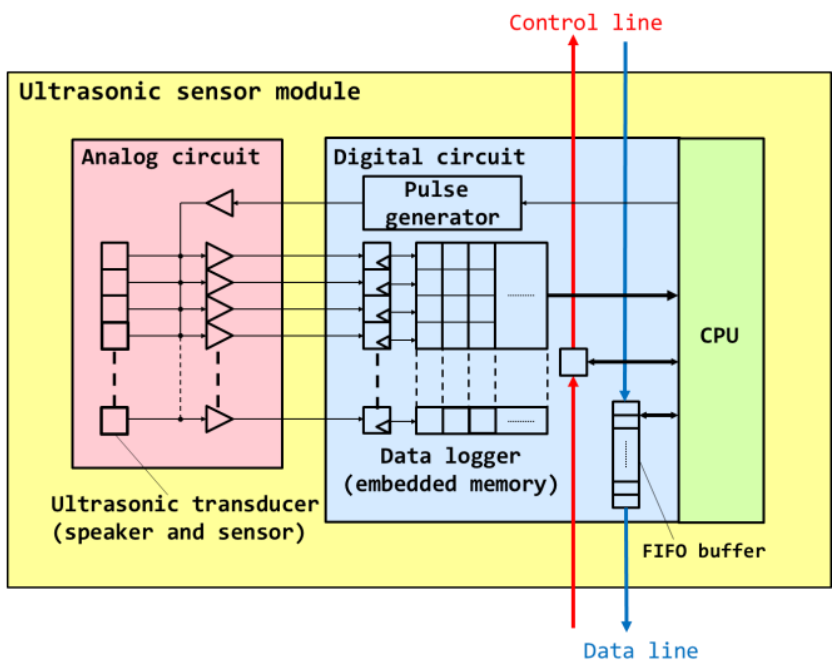

Fig. 3. Ultrasonic Sensor Module

\subsection{Structure of Ultrasonic Sensor Module}

Fig. 3 shows the structure of the ultrasonic sensor module in the proposed array. The module is composed of analog circuit, digital circuit and an embedded processor. The ultrasonic transducer operates as both speaker and sensor is used. Communication among modules and the host computer is performed on the control line and the data line. In the control line, shift register is constructed and the command from the host computer is shifted out from the tail module to the top module. After the command is received at each module, the processed data is sent from the top module to the tail module in the data line. Each module has FIFO buffer because the size of the data changes due to the number of the connected modules. When signal indicating the beginning of measuring is received from the host computer via the control line, the processer in the module drives the pulse generator in the digital circuit. The generated pulse is amplified in the analog circuit and transmitted in the ultrasonic transducer. The reflected wave at the stimulated part of the measured object is received in the ultrasonic transducer. The received signal is amplified and captured in the flip-flop at a certain time. The captured data is saved into the embedded memory like Fig. 2. Using the saved data, distance from the transducer to the stimulated part is calculated in the processer and the calculated data is saved in the FIFO buffer.

\section{Design of Daisy-chain Connection}

\subsection{Whole of Proposed Structure}

Fig. 4 shows the whole of the proposed ultrasonic sensor array. All modules are same structures and arbitrary 
number of modules can be connected between the tail module and the top module. Each module is connected by the control line and the data line. In the top module, the output of the control line is input into the data line. So the module can recognize itself as the top module.

\subsection{Execution of Sensor Array}

Fig. 5 shows the execution of the proposed sensor array. We explain the operation of the proposed sensor array when two modules that are composed of the tail module and the top module are connected.

Host computer sending command in Fig. 5(a): Command to drive each module is sent form the host computer. This command have two counters. The one of them is the counter that indicates the number of the modules that finish operating (operated counter). The other is the counter that indicates the number of the modules that finish sending processed data (sent counter).

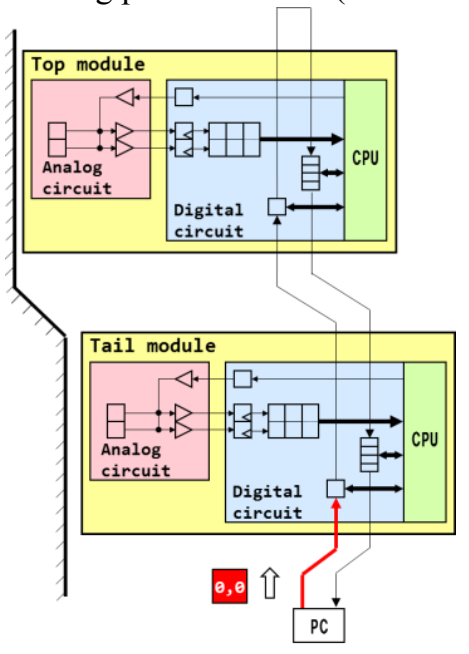

(a) Host computer sending command

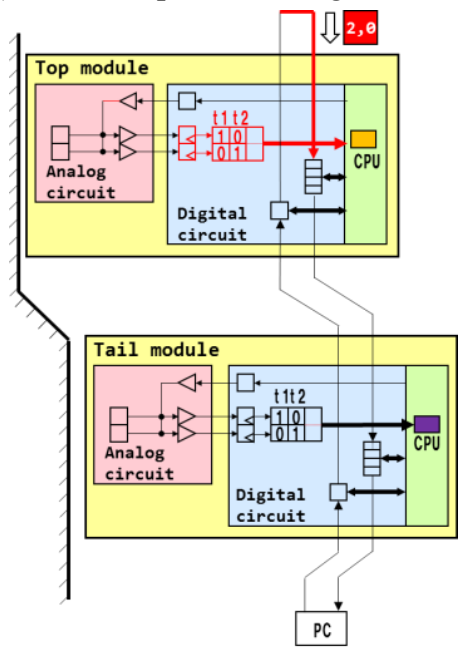

(d) Command turned back

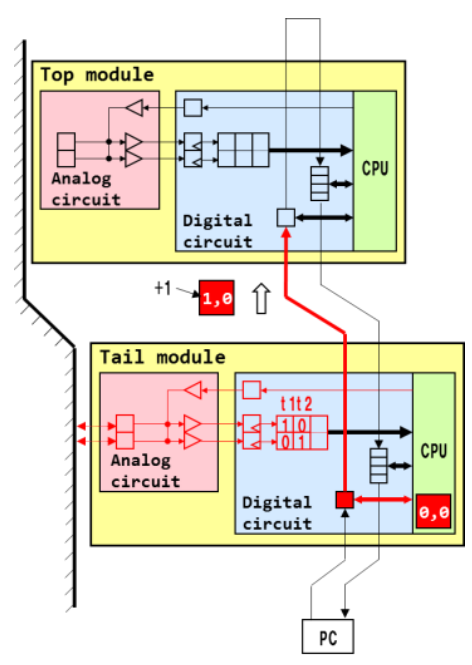

(b) Tail module receiving command

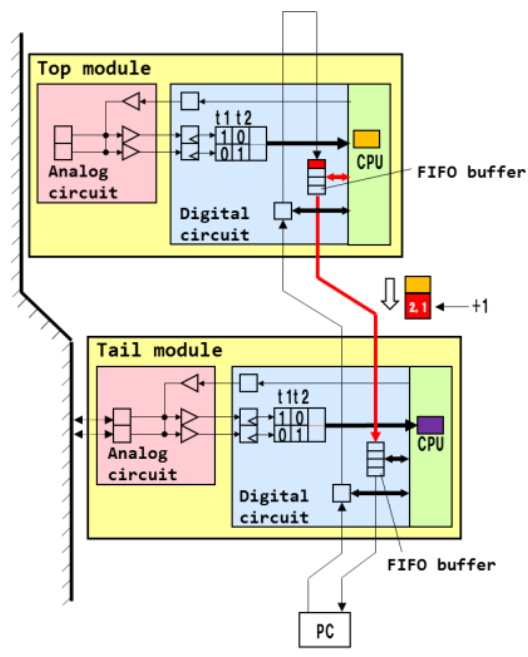

(e) Top module sending distance data

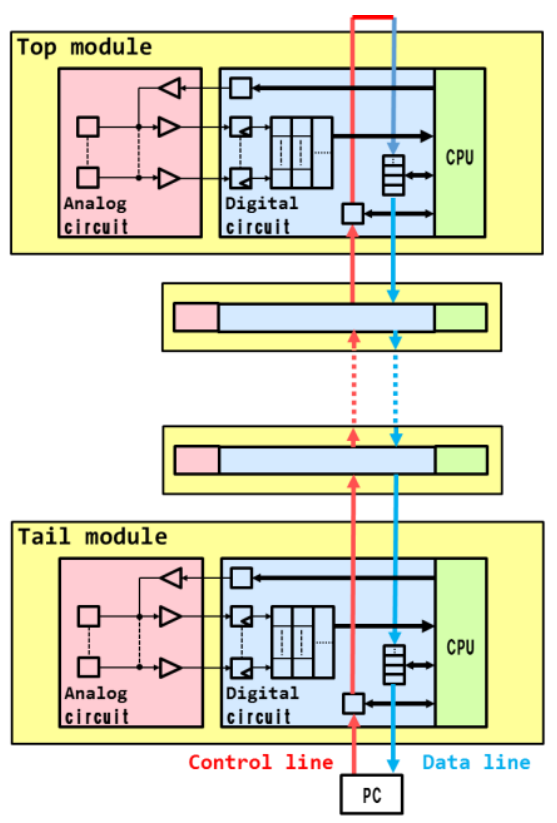

Fig. 4. Whole of Sensor Array

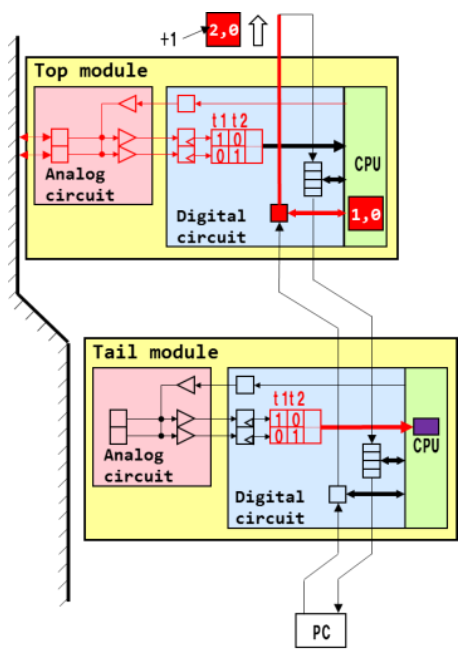

(c) Top module receiving command

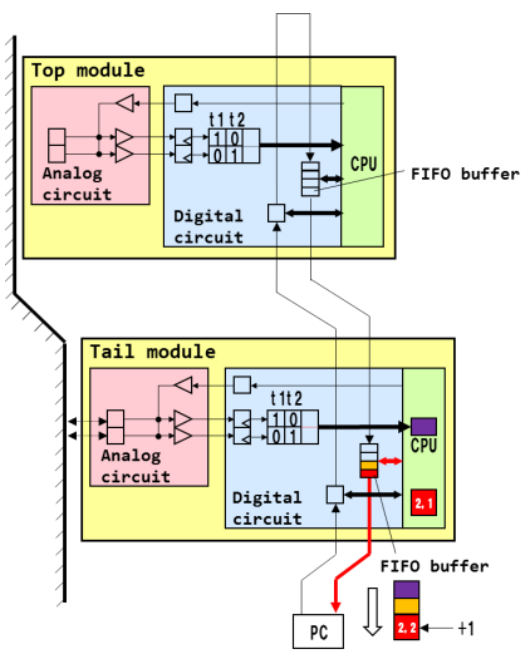

(f) Tail module sending distance data

Fig. 5. Execution Snapshot of Proposed Ultrasonic Sensor Array 
Tail module receiving command in Fig. 5(b): When the command is received in the tail module, the module starts measurement. The command is counted up in the operated counter and sent to the next module.

Top module receiving command in Fig. 5(c): Top module also starts measurement when the command is received form the next module. The command is counted up in the operated counter and output outside. In the tail module, distance is calculated and saved into the memory.

Command turned back in Fig. 5(d): In the top module, distance is calculated and saved into the memory, too. The command that is output from the top module is input into the top module again via an outside cable and turned back to the host computer through modules.

Top module sending distance data in Fig. 5(e): The module can recognize itself as the top module when the command turned back to the host is input into the FIFO buffer. Then the top module sends the calculated distance data with the command to the next module. When the top module sends them, the command is counted up in the sent counter.

Tail module sending distance data in Fig. 5(f): When the tail module receives the command and distance data, they are saved into the FIFO buffer. The tail module adds distance data to the data saved into the FIFO buffer and sends them to the host computer. When they are sent to the host computer, the command is counted up in the sent counter. When the host computer confirm that the operated count in the command marches the sent count, the host computer can check the errors in each module.

\section{Preliminary Evaluation}

\subsection{Communication Data}

Fig. 6 shows the structure of the data used in the communication among the host computer and the modules. The communication from the host computer to the top module (upstream) is performed by only the operated counter. The data size of the counter is set on 1 byte. So the number of module that is up to 255 can be counted. The communication form the top modules to the host computer (downstream) is performed by two counters (operated counter and sent counter) and distance information calculated by each module. The data size of each counter is 1 byte. The data size of the distance data is depending on the number of sensors installed on the module and also varies by the number of the connected modules.

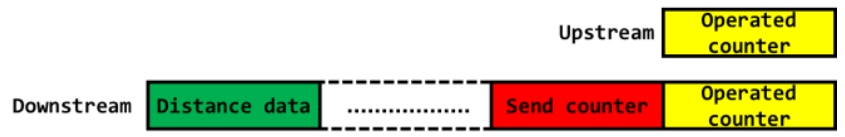

Fig. 6. Communication Data

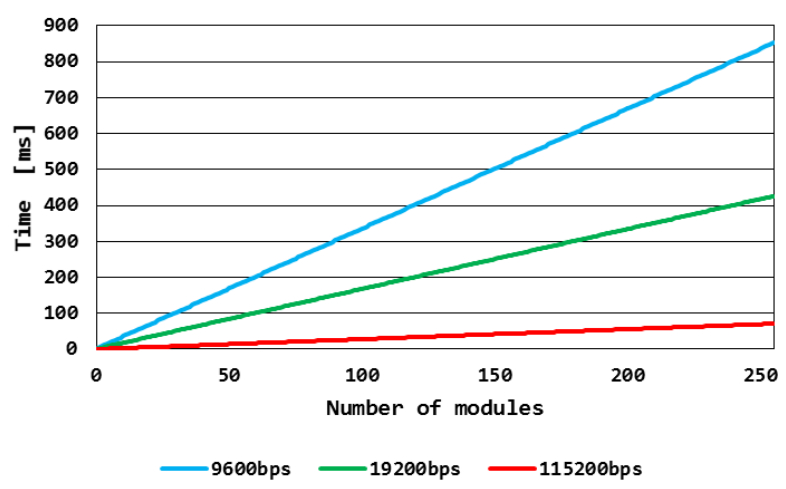

Fig. 7. Relation between Number of Modules and Communication Time

\subsection{Estimation of Communication Time}

Total time $t$ needed to send the command from the host computer and collect distance information from each module is calculate by following expression.

$$
t[\mathrm{sec}]=\frac{24+b c}{a}
$$

Where $a$ is transfer rate [bit/sec], $b$ is the data size that is required by the single module [bit] and $c$ is the number of the modules. Fig. 7 shows the relation of the number of the modules and the communication time if the data size $b$ is set in 32 bit. The more number of the connected modules increases, the more time is needed to send the command and collect distance information from each module. So users and designers have to adjust suitable transfer rate according to the number of the modules and the purpose of the measuring. If the target objects is moving and many modules are needed, the transfer rate would be set in high.

\section{Conclusions}

We have proposed the ultrasonic sensor array with scalability and flexibility to any objects. In this proposed array, the modules equipped with intelligent units composed of analog circuit, digital circuit and a processor. The modules that can be adhered to the measured objects are connected by daisy-chain fashion. This structure enables us to measure objects with arbitrary size and shape. As a preliminary evaluation, the delay was estimated because the 
communication among the host and each module is performed by serial communication. As a result, transfer rate have to be adjusted according to the purpose of the measuring and the number of the connected modules.

This work is at the stage of the design and preliminary evaluation of the proposed ultrasonic sensor array. As further work, we will develop the prototype and value the delay. In addition, we will improve the communication speed, user interface and reliability.

\section{References}

(1) Yuko Yamamoto, Seiichi Kawanami, Naoto Kawase, et al : "Technology of Flexible Thin-film Ultrasonic testing Sensor with High Temperature Resistance", Mitsubishi Heavy Industries Technical Review Vol. 52, No. 4, pp. 111-117, 2015

(2) Ira O. Wygant, Xuefeng Zhuang, David T. Yeh, Omer Oralkan, A. Sanli Ergun, Mustafa Karaman, Butrus T. Khuri-yakub : "Integration of 2D CMUT arrays with front-end electronics for volumetric ultrasound imaging", IEEE Transactions on Ultrasonic, Ferroelectrics, and Frequency Control, Vol. 55, No. 2, pp. 327-342, 2008

(3) Anshuman Bhuyan, Jung Woo Choe, Byung Chul Lee, Ira O. Wygant, Amin Nikoozadeh, Ömer Oralkan, Butrus T. Khuri-Yakub : "Integrated Circuits for Volumetric Ultrasound Imaging With 2-D CMUT Arrays", IEEE Transactions on Biomedical Circuits and Systems, Vol. 7, No. 6, pp. 796-804, 2013

(4) Yuichi Morita, Kono Sota, and Akira Yamawaki : "Proposal of an Ultrasonic Sensor Array with Flexible and Scalable Organization", Proceedings of the TwentyThird International Symposium on Artificial Life and Robotics 2018, pp. 381-384, 2018 